A. G. Earnest

Nagoya Math. J.

Vol. 93 (1984), 27-38

\title{
REPRESENTATION OF SPINOR EXCEPTIONAL INTEGERS BY TERNARY QUADRATIC FORMS
}

\author{
A. G. EARNEST
}

\section{§ 0 . Introduction}

The existence and basic properties of what are now referred to as spinor exceptional integers for a genus of integral ternary quadratic forms were first observed in the 1950's by Jones and Watson [7] and Kneser [8] in the context of indefinite forms. The study of these integers and their generalizations has been undertaken by a number of authors in recent years, and has contributed significantly to the understanding of representation properties unique to ternary forms. In this direction, the present author proved in a previous paper [4] that if $c$ is a primitive spinor exceptional integer for a genus of integral ternary quadratic forms and $f$ is some form in this genus, then a form in the spinor genus of $f$ primitively represents $c$ if and only if $f$ primitively represents an integer of the type $c t^{2}$, for some odd positive integer $t$, relatively prime to the discriminant $d$, which satisfies the condition that the Jacobi symbol $(-c d / t)$ equals 1 .

In Section 1 of the present paper we prove that, in the context described in the previous paragraph, the Jacobi symbol $(-c d / t)$ precisely determines which integers of the type $c t^{2}$ are primitively represented by any given spinor genus. As the spinor genus and isometry class coincide for indefinite ternary forms, this result directly gives information regarding the integers represented by such a form. However, since a spinor genus of positive definite ternary forms can contain many isometry classes, these definite forms can fail to represent values represented by their spinor genus. We prove in Section 2 that a positive definite ternary form can fail to primitively represent at most a finite number of integers of the type $c t^{2}$ for which the value of the Jacobi symbol $(-c d / t)$ is compatible for primitive representation by the spinor genus of the form. In

Received August 2, 1982. 
Section 3 we drop the assumption that $c$ is a primitive spinor exceptional integer for the genus, and use the results of the preceding sections to produce an infinite family of integers of the type $c t^{2}$ primitively represented by the form under the assumption only that $c$ is squarefree.

The theorem quoted in the first paragraph of this introduction has been generalized by Benham and Hsia [1] to the setting of quadratic lattices over the rings of integers of arbitrary algebraic number fields. With necessary notational changes, the theorem carries over to that context with the Jacobi symbol $(-c d / t)$ being replaced by an appropriate Artin symbol. The results of Section 1 of the present paper can be phrased in this generality as well. However, as the remainder of the paper deals with the classical case of forms over the rational integers, we prefer to adopt that context consistently throughout.

We will use the geometric language of quadratic spaces and lattices, with unexplained terminology and notation following that of O'Meara's book [9]. We work always within a fixed nondegenerate quadratic space $(V, Q)$ of dimension 3 over the field $\boldsymbol{Q}$ of rational numbers. We denote by $B($,$) the symmetric bilinear form related to Q$ by $Q(x+y)=Q(x)$ $+Q(y)+2 B(x, y)$ for $x, y \in V$. The lattices $L$ considered will be $Z$-lattices on $V$ (i.e., finitely generated $Z$-modules contained in $V$ whose vectors span $V$ ) with the additional assumptions that $Q(x) \in Z$ for all $x \in L$ and the ideal generated by all such values equals $Z$. Such lattices correspond (as in $[9 ; \S 41 \mathrm{c}]$ ) to primitive integral ternary quadratic forms. For a lattice $L$ having $Z$-basis $\left\{x_{1}, x_{2}, x_{3}\right\}$, define the discriminant $d L$ of $L$ to be $4 \operatorname{det}\left(B\left(x_{i}, x_{j}\right)\right)$. The discriminants of all lattices considered here will be the same, with their common value denoted simply by $d$.

The set of all integers represented (primitively) by a lattice $L$ will be denoted by $Q(L)\left(Q^{*}(L)\right.$, respectively). For an integer $c$ and a genus or spinor genus $X$ of lattices, we will write $c \in Q(X)$, or $c \in Q^{*}(X)$, if there exists at least one lattice $K$ in $X$ such that $c \in Q(K)$, or $c \in Q^{*}(K)$, respectively.

The integer $c$ is a spinor exceptional integer for a genus $G$ of ternary lattices if $c \in Q(G)$ but there exists at least one spinor genus $S$ contained in $G$ such that $c \notin Q(S) ; c$ is a primitive spinor exceptional integer if $c \in$ $Q^{*}(G)$ but there exists at least one $S$ such that $c \notin Q^{*}(S)$. The spinor exceptional integers for a genus can be determined by local information using the necessary and sufficient conditions of Schulze-Pillot [11], which 
are summarized in (3.1)-(3.3) of this paper. These integers are truly exceptional in the sense that they occur only in finitely many square classes for any given genus. However, within any such square class all but at most finitely many of the integers are spinor exceptional. In fact, if $c$ is a spinor exceptional integer for the genus $G$, then so is $c t^{2}$ for all odd integers $t$ relatively prime to the discriminant (see e.g. [14]). Further properties of these integers will be summarized, with pertinent references, as they are needed. It should be noted that what we refer to here for the sake of clarity and consistency with the current literature as "primitive spinor exceptional integers" are called simply "exceptional integers" in the books of Watson [14] and Cassels [2].

\section{$\S 1$. Representation by the spinor genus}

The main result of this section is:

THEOREM 1. Let c be a primitive spinor exceptional integer for a genus $G$ of ternary quadratic lattices, and let $S$ be a spinor genus contained in $G$. Let $s$ be a fixed positive integer relatively prime to $2 d$ for which $c s^{2} \in Q^{*}(S)$. If $t$ is any positive integer relatively prime to $2 d$, then $c t^{2} \in Q^{*}(S)$ if and only if $(-c d / s)=(-c d / t)$ (interpreting here $(a / b)=+1$ in case $b=1)$.

As the proof of this theorem rests on the key Proposition 1.2 of the previous paper [4], we will first recall the setting and content of that proposition and then present a slightly modified version of its proof, for which I am indebted to Dr. Schulze-Pillot [12]. The revised proof has the advantage of eliminating the need for the approximation of local bases, replacing this step by a more standard construction.

For the statement of the proposition, we use the notation of idèles and split rotations as described in [9]. In particular, $J_{V}$ will denote the split rotation group of $V$ and $J_{Q}$ the full idèle group of $\boldsymbol{Q}$. Let $\theta: J_{V} \rightarrow$ $J_{Q} / J_{Q}^{2}$ be the mapping obtained by applying the local spinor norm mappings to each coordinate of an element of $J_{V}$. For an integer $t$, define an idèle $j(t)$ coordinatewise by

$$
j(t)_{p}= \begin{cases}1 & \text { if } \operatorname{ord}_{p} t \text { is even, or } p=\infty \\ p & \text { if } \operatorname{ord}_{p} t \text { is odd. }\end{cases}
$$

Proposition 1. Let $L$ be a ternary quadratic lattice and let $c$ be an integer. If $c t^{2} \in Q^{*}(L)$ for some integer $t$ with $\operatorname{gcd}(t, 2 d)=1$, then $c \in Q^{*}(\Sigma L)$ for some $\Sigma \in J_{V}$ with $\theta(\Sigma)=j(t) J_{Q}^{2}$. 
Proof. Let $v$ be a primitive vector of $L$ with $Q(v)=c t^{2}$. If $p$ is a prime dividing $t$, then $p \times 2 d$ by hypothesis. It follows from the local theory that for each such $p$, there exists a $Z_{p}$-basis $x_{1}^{(p)}, x_{2}^{(p)}, x_{3}^{(p)}$ for $L_{p}$ for which the matrix

$$
\left(B\left(x_{i}^{(p)}, x_{j}^{(p)}\right)\right)=\left(\begin{array}{ll}
0 & 1 \\
1 & 0
\end{array}\right) \perp\langle-d\rangle, \quad \text { and } \quad v=c t^{2} x_{1}^{(p)}+\frac{1}{2} x_{2}^{(p)} .
$$

Consider the lattice $K$ on $V$ having localizations

$$
K_{p}=\left\{\begin{array}{l}
Z_{p}\left(t x_{1}^{(p)}\right)+Z_{p}\left(t^{-1} x_{2}^{(p)}\right)+Z_{p} x_{3}^{(p)}, \text { if } p \mid t \\
L_{p}, \text { if } p \nmid t .
\end{array}\right.
$$

It is easily checked that $t^{-1} v$ is a primitive vector of $K$. For $p \mid t, K_{p}=$ $\sigma_{p} L_{p}$ where $\sigma_{p} \in O^{+}\left(V_{p}\right)$ is defined by $\sigma_{p}\left(x_{1}^{(p)}\right)=p^{s_{p}} x_{1}^{(p)}, \sigma_{p}\left(x_{2}^{(p)}\right)=p^{-s_{p}} x_{2}^{(p)}$ and $\sigma_{p}\left(x_{3}^{(p)}\right)=x_{3}^{(p)}$ where $s_{p}=\operatorname{ord}_{p} t$. By [9; 55:1], the spinor norm of $\sigma_{p}$ is $p^{s} \dot{Q}_{p}^{2}$. Define the element $\Sigma$ of $J_{V}$ by

$$
\Sigma_{p}= \begin{cases}\sigma_{p} & \text { if } p \mid t \\ \operatorname{Id}_{p} & \text { if } p \nmid t .\end{cases}
$$

Then $\Sigma L=K$ and $\theta(\Sigma)=j(t) J_{Q}^{2}$.

Before proving Theorem 1, it is helpful to recall some terminology and basic results concerning spinor exceptional integers. If $c$ is a primitive spinor exceptional integer for a genus $G$, then $c$ is primitively represented by exactly half of the spinor genera of $G$; thus, we say that the set of spinor genera of $G$ is partitioned into two c-half-genera. This partitioning can be described quite precisely. To do so, consider the subgroup $N_{c}$ of $J_{Q}$ defined by

$$
N_{c}=\left\{j \in J_{Q}:\left(j_{p},-c d\right)_{p}=+1 \text { for all primes } p\right\},
$$

where $(,)_{p}$ denotes the Hilbert symbol. When $c$ is a primitive spinor exceptional integer for $G$, the group index $\left[J_{Q}: P_{D} N_{c} J_{Q}^{L}\right]$ equals 2 (here $L$ is a lattice in $G$; $J_{Q}^{L}$ depends only upon $G$ ), and two spinor genera $S$ and $T$ of $G$ lie in the same $c$-half-genus (i.e., both primitively represent $c$ or both fail to do so) if and only if $S=\Sigma T$ for some $\Sigma \in J_{V}$ with $\theta(\Sigma)$ $\subseteq P_{D} N_{c} J_{Q}^{L}$. Note that the subgroup $N_{c}$ depends only upon the square class of $c$; thus, the splitting of $G$ into $c t^{2}$-half-genera for any $t$ with $\operatorname{gcd}(t, 2 d)=1$ is the same as the splitting into $c$-half-genera. For the particular idèles $j(t)$ introduced earlier, containment in the index 2 
subgroup $P_{D} N_{c} J_{Q}^{L}$ can be described as follows:

Lemma 1 [4; Lemma 2.1]. Suppose $c$ is a primitive spinor exceptional integer for $G$ and $t$ is a positive integer with $\operatorname{gcd}(t, 2 d)=1$. Then $j(t)$ $\in P_{D} N_{c} J_{Q}^{L}$ if and only if $(-c d / t)=+1$.

Proof of Theorem 1. Since $(t, 2 d)=1, G$ primitively represents $c t^{2}$. So there is some spinor genus, say $T$, in $G$ for which $c t^{2} \in Q^{*}(T)$. $S$ primitively represents $c t^{2}$ if and only if $S$ is in the same $c t^{2}$-half-genus, or, equivalently, the same $c$-half-genus, as $T$. According to Proposition 1 and Lemma $1, S$ and $T$ are in the same $c$-half-genus if and only if $(-c d / t)=(-c d / s)$.

If $L$ is an indefinite ternary lattice, then the isometry class and spinor genus of $L$ coincide as a consequence of the Stong Approximation Theorem for Rotations [9; 104: 4]. Hence, we have:

Corollary. Let $L$ be an indefinite ternary lattice, and let $c$ be a primitive spinor exceptional integer for gen $(L)$. Let $s$ be a fixed positive integer relatively prime to $2 d$ for which $c s^{2} \in Q^{*}(L)$. If $t$ is any positive integer relatively prime to $2 d$, then $c t^{2} \in Q^{*}(L)$ if and only if $(-c d / t)=$ $(-c d / s)$.

In light of this result, consider the problem of determining whether a particular integer is primitively represented by an indefinite ternary lattice. Even for small values, such a problem is quite formidable from a computational standpoint since no bounds can be imposed on the values of the indeterminates required to produce such a representation.

By local computation alone it is routine to determine whether the given integer satisfies the necessary condition that it be primitively represented by the genus of the lattice. If this necessary condition is met, it can be further determined by the local conditions to be given in (3.1)(3.3) whether the integer in question is a primitive spinor exceptional integer for the genus. If not, the issue is settled, since the integer is then primitively represented by every lattice in the genus. So only the integers in a square class of primitive spinor exceptional integers require further analysis. For the integers in such a square class, the above corollary shows that the question can be resolved by producing a primitive representation of any integer in the square class.

We illustrate the method of the preceding paragraph by analyzing 
an example considered by Siegel in $[13 ;$ p. 52].

EXAmple 1. Consider the lattices $L$ and $K$ corresponding to the forms $f=x^{2}-2 y^{2}+64 z^{2}$ and $g=(2 x+z)^{2}-2 y^{2}+16 z^{2}$, respectively. $L$ and $K$ are representatives of the only two isometry classes in their genus. Applying the criteria given in (3.1)-(3.3), it can be shown that the only primitive spinor exceptional integers for this genus are the odd squares. To see which odd squares are represented by $L$ and which by $K$, it suffices to observe that $1 \in Q^{*}(L)$ and use the above corollary. In this case, $c=1$ and $d=-2^{9}$; so $(-c d / t)=(2 / t)$. Hence, for an odd positive integer $t, t^{2}$ is primitively represented by $L$ if and only if $t \equiv \pm 1(\bmod 8)$, and $t^{2}$ is primitively represented by $K$ if and only if $t \equiv \pm 3(\bmod 8)$.

\section{$\S 2$. Representation of spinor exceptional integers by positive defi- nite forms}

We turn our attention in this section to the positive definite case, where the added difficulty that a particular spinor genus can contain many isometry classes arises. In general when it is known that the spinor genus of a lattice $L$ represents some integer $c$, there are standard methods which can be used to draw conclusions concerning values of the type $c t^{2}$ represented by $L$ itself. For example, if $p$ is any prime at which $V_{p}$ is isotropic, then it can be shown that $L$ represents some integer of the type $c p^{2 k}$.

It has been conjectured by Cassels [3] that in fact a positive definite ternary lattice represents all but at most finitely many of the integers represented by its spinor genus. While this conjecture as stated is false, it is very likely that the analogous statement for primitive representations is true. In this direction, the main theorem of this section is:

THEOREM 2. Let $L$ be a positive definite ternary lattice and let $c$ be a primitive spinor exceptional integer for gen $(L)$. Then $L$ primitively represents all but at most finitely many of the integers of the type $c t^{2},(t, 2 d)=1$, which are primitively represented by $\operatorname{spn}(L)$.

For the proof of this result we will follow the method of Peters [10], who uses techniques of Linnik and Malyshev to prove a partial and conditional analogue for positive definite ternary lattices to the wellknown result (see e.g. [2]) that a positive definite lattice in 4 or more variables primitively represents all sufficiently large integers which are 
primitively represented by its genus.

For the remainder of this section $L$ will denote a fixed positive definite ternary lattice. If $L$ has $Z$-basis $\left\{x_{1}, x_{2}, x_{3}\right\}$, let $d_{2}$ be the greatest common divisor of the $2 \times 2$-subdeterminants of the matrix $\left(2 B\left(x_{i}, x_{j}\right)\right)$. Let $D=$ $16 d / d_{2}^{2}$ (with $d$ as in Section 0). The relationship between $D$ and the norm form of a quaternion order associated to $L$ is described in [10; p. 68]. For a lattice $K$ and an integer $a$ we denote the number of primitive vectors $x$ in $K$ with $Q(x)=a$ by $A^{*}(a, K)$. Finally, for a positive integer $\Delta$, let $H(\Delta)$ be the number of proper equivalence classes of primitive positive definite binary quadratic forms of discriminant $\Delta$.

With these notations established, we begin by isolating from [10; Section 5] the crucial step in the application of the methods of Linnik and Malyshev:

Proposition 2. Let $p$ be a prime for which $p \times 2 D$ and $V_{p}$ is isotropic, and let $C$ be a collection of integers. If there exist constants $\ell_{0} \in N$ and $\alpha$, depending on $p$ and $L$, such that for each $c \in C$,

$$
A^{*}\left(c p^{2 \ell}, L\right)>\alpha H(2 d c)
$$

holds for some integer $\ell$ (which depends on $c$ ) between 0 and $\ell_{0}$, then $L$ primitively represents all but at most finitely many $c \in C$ satisfying $(-D c / p)$ $=1$.

To relate the hypothesis of Proposition 2 to spinor genus representations, it is necessary to recall the definitions and basic properties of the weight of representations within a genus or spinor genus. If $L=L_{1}, \cdots$, $L_{h}$ are representatives for the distinct isometry classes in gen $(L)$, then for an integer $a$ define

$$
A^{*}(a, \operatorname{gen}(L))=w^{-1} \sum_{i=1}^{n} A^{*}\left(a, L_{i}\right)\left|O\left(L_{i}\right)\right|^{-1}
$$

where

$$
w=\sum_{i=1}^{h}\left|O\left(L_{i}\right)\right|^{-1}
$$

Moreover, if $K \in$ gen $(L)$ and $K=K_{1}, \cdots, K_{s}$ are representatives for the distinct isometry classes in spn $(K)$, define

$$
A^{*}(a, \operatorname{spn}(K))=z(K)^{-1} \sum_{j=1}^{s} A^{*}\left(a, K_{j}\right)\left|O\left(K_{j}\right)\right|^{-1}
$$

where 


$$
z(K)=\sum_{j=1}^{s}\left|O\left(K_{j}\right)\right|^{-1} .
$$

From (2.2) and (2.3), one can immediately derive

$$
w A^{*}(a, \operatorname{gen}(L))=\sum_{k=1}^{g} z\left(M_{k}\right) A^{*}\left(a, \operatorname{spn}\left(M_{k}\right)\right),
$$

where $M_{1}, \cdots, M_{g}$ are representatives of the distinct spinor genera in gen $(L)$. A basic theorem of Kneser [8] shows that either the values $A^{*}\left(a, \operatorname{spn}\left(M_{k}\right)\right)$ are equal for all $k=1, \cdots, g$, or there are exactly two such values, each taken on by exactly half of the $k$ 's (see also [5]). In particular, if $c$ is a primitive spinor exceptional integer for gen $(L)$ and $c \in Q^{*}(\operatorname{spn}(L))$, then $A^{*}(c, \operatorname{spn}(K))=A^{*}(c, \operatorname{spn}(L))$ for every $K$ in the $c$ half-genus of $L$.

Proof of Theorem 2. Let $T$ denote the set of all positive integers $t$ which are relatively prime to $2 d$ and for which $c t^{2} \in Q^{*}(\operatorname{spn}(L))$. By [10; Satz 3.1] there exists a constant $\beta$, depending only on gen $(L)$, for which

$$
A^{*}\left(c t^{2}, \operatorname{gen}(L)\right)>\beta H\left(2 d c t^{2}\right)
$$

holds for all $t \in T$. With the notations of the previous paragraph, we obtain from (2.4) and (2.5) that

$$
\sum_{k=1}^{g} z\left(M_{k}\right) A^{*}\left(c t^{2}, \operatorname{spn}\left(M_{k}\right)\right)>w \beta H\left(2 d c t^{2}\right)
$$

holds for all $t \in T$. Since $c t^{2}$ is a primitive spinor exceptional integer for gen $(L)$ for each $t \in T$, half of the $A^{*}\left(c t^{2}, \operatorname{spn}\left(M_{k}\right)\right)$ are equal zero, and the other half are all equal to the same nonzero value. Since $A^{*}\left(c t^{2}, \operatorname{spn}(L)\right)$ $\neq 0$ for $t \in T$, if follows from (2.6) that

$$
A^{*}\left(c t^{2}, \operatorname{spn}(L)\right)>r H\left(2 d c t^{2}\right)
$$

holds for all $t \in T$, where $\gamma$ is a constant depending on gen $(L)$. It follows from (2.3) that there exists a constant $\delta$, depending on $L$, such that for each $t \in T$ there exists a lattice $K(t) \in \operatorname{spn}(L)$ for which

$$
A^{*}\left(c t^{2}, K(t)\right)>\delta H\left(2 d c t^{2}\right) .
$$

Now let $p$ be a prime for which $V_{p}$ is isotropic and $(-D c / p)=1$. Let $K_{1}, \cdots, K_{s}$ be representatives of the distinct isometry classes in spn $(L)$. By $[9 ; 104: 5]$, there exists a natural number $\ell_{0}$ (depending on $p$ and $L$ ) such that $p^{\ell_{0}} K_{i} \subseteq L$ for all $i=1, \cdots, s$. In particular, every vector $x$ 
counted in $A^{*}\left(c t^{2}, K(t)\right)$ in (2.8) satisfies $p^{\ell_{0}} x \in L$. So for each such $x$, there exists an $\ell$ between 0 and $\ell_{0}$ for which $p^{\ell} x$ is a primitive vector of $L$. From (2.8) we obtain that there exists a constant $\alpha$ depending on $p$ and $L$ such that, for each $t \in T$, there exists an integer $\ell$ between 0 and $\ell_{0}$ for which

$$
A^{*}\left(c t^{2} p^{2 \ell}, L\right)>\alpha H\left(2 d c t^{2}\right)
$$

holds. The theorem now follows by applying Proposition 2 to the collection $C$ of integers of the type $c t^{2}, t \in T$.

Remark. The removal of the assumption that $c$ is a primitive spinor exceptional integer for gen $(L)$ in Theorem 2 would necessitate consideration of integers $a$ for which $A^{*}(a, \operatorname{spn}(K))$ takes on two different values, say $\Lambda_{1}$ and $\Lambda_{2}$, as $K$ ranges through representatives for the spinor genera in gen $(L)$. From this point of view, it would be of interest to know to what extent $\Lambda_{1}$ and $\Lambda_{2}$ could differ. Specifically, suppose that $c$ and $L$ are fixed, with $c \in Q^{*}(\operatorname{gen}(L))$, and write $\Lambda_{K}(t)=A^{*}\left(c t^{2}, \operatorname{spn}(K)\right)$ and $\Lambda_{M}(t)$ $=A^{*}\left(c t^{2}, \operatorname{spn}(M)\right)$, for lattices $K$ and $M$ in gen $(L)$. If the ratio $\Lambda_{K}(t) / \Lambda_{M}(t)$ could be bounded independently of $t$, then the argument of the proof of Theorem 2 could be extended to encompass the integers of the type $c t^{2}$ for such $c$.

\section{§3. Representation of $c t^{2}$ for squarefree integers $c$}

The results of this section were motivated by a conjecture of $\mathrm{B}$. W. Jones [6] that if $f$ is a primitive ternary quadratic form which primitively represents a square relatively prime to twice the discriminant of $f$, then $f$ primitively represents an infinite number of squares of primes. Using the results of the first two sections of this paper, we will show that in fact there is a choice of $\varepsilon= \pm 1$ such that under the above assumptions $f$ primitively represents all but at most finitely many of all squares $t^{2}$ with $(t, 2 d)=1$ which satisfy $(-d / t)=\varepsilon$.

This is a special case of the following result for integers of the type $c t^{2}$ where $c$ is only assumed to be squarefree.

THEOREM 3. Let $L$ be a ternary quadratic lattice and let c be a squarefree integer which is primitively represented by the genus of $L$. Then, for at least one choice of $\varepsilon= \pm 1, L$ primitively represents all but at most finitely many integers of the type $c t^{2}$ with $(t, 2 d)=1$ which satisfy $(-c d / t)=\varepsilon$. 
If $c$ is a primitive spinor exceptional integer for gen $(L)$, then Theorem 3 follows immediately either from the Corollary to Theorem 1 (for the indefinite case) or from Theorem 2 (for the definite case). So it remains to study the case that $c$ is not a primitive spinor exceptional integer. For this purpose, it is necessary to use the precise local conditions which characterize such integers, as determined by Schulze-Pillot [11]. We will state these conditions here for reference.

Let $c \in Q(\operatorname{gen}(L)), c \neq 0$. For a rational prime $p$, let $x$ be a fixed vector of $L_{p}$ for which $Q(x)=c$. Denote by $\theta\left(L_{p}, c\right)$ the subgroup of $\dot{\boldsymbol{Q}}_{p}$ which is generated by all $\alpha \in \dot{\boldsymbol{Q}}_{p}$ for which there exists a $\sigma \in O^{+}\left(V_{p}\right)$ with $\alpha \in \theta(\sigma)$ and $x \in \sigma\left(L_{p}\right)$. Let $\left(N_{c}\right)_{p}=\left\{\beta \in \dot{Q}_{p}:(\beta,-c d)_{p}=1\right\}$, and let $J_{L}$ be the subgroup of $J_{V}$ consisting of those $\Sigma$ for which $\Sigma_{p} \in O^{+}\left(L_{p}\right)$ for all finite primes $p$. With other notations as in Section 1, necessary and sufficient conditions under which $c$ is a spinor exceptional integer for gen $(L)$ are as follows [11; Satz 2]:

$$
\begin{gathered}
-c d \notin \dot{\mathbf{Q}}^{2} \\
\theta\left(J_{L}\right) \subseteq N_{c}
\end{gathered}
$$

and

$$
\theta\left(L_{p}, c\right)=\left(N_{c}\right)_{p}
$$

where (3.3) must hold for all rational primes $p$.

Proof of Theorem 3. As noted above, it suffices to consider the case that $c$ is not a primitive spinor exceptional integer for gen $(L)$. So assume that at least one of the conditions (3.1)-(3.3) is violated. It is shown by Peters [10] that the hypotheses of Proposition 2 are satisfied by the collection $C$ consisting of all integers for which either (3.1) or (3.2) is violated. Thus, we may further restrict to the case that (3.1) and (3.2) are satisfied for $c$, but (3.3) is violated.

If $(-c d) \in \dot{Q}_{p}^{2}$ for some prime $p$, then $\theta\left(L_{p}, c\right)=\left(N_{c}\right)_{p}$ holds as a consequence of the containments $\left(N_{c}\right)_{p} \subseteq \theta\left(L_{p}, c\right) \subseteq \dot{\boldsymbol{Q}}_{p}$. So we assume for the remainder of the proof that $(-c d) \notin \dot{\boldsymbol{Q}}_{p}^{2}$ and that $\theta\left(O^{+}\left(L_{p}\right)\right) \subseteq\left(N_{c}\right)_{p}$. Under these assumptions, the conditions under which the equality $\theta\left(L_{p}, c\right)=\left(N_{c}\right)_{p}$ is violated for an odd prime $p$ are enumerated in [11; Satz 3]. A check of the four cases of that theorem shows that these conditions never hold when $\operatorname{ord}_{p} c=0$ or 1 . As we are assuming that $c$ is squarefree, these are the only possibilities for $\operatorname{ord}_{p} c$ here. We conclude that under our 
hypotheses and the restrictions of the previous paragraph, the equality $\theta\left(L_{p}, c\right)=\left(N_{c}\right)_{p}$ holds for all odd primes $p$.

It remains to consider those integers $c$ for which $\theta\left(L_{2}, c\right) \neq\left(N_{c}\right)_{2}$. For such integers, we will show that there exists a sublattice $L^{\prime}$ of $L$ for which $c \in Q^{*}\left(\operatorname{gen}\left(L^{\prime}\right)\right)$ and (3.1)-(3.3) are satisfied by $c$ and $L^{\prime}$. Moreover, $L^{\prime}$ is constructed in such a way that $\left(L^{\prime}\right)_{p}=L_{p}$ for all odd primes $p$. So if $x$ is a primitive vector of $L^{\prime}$ with $Q(x)=c t^{2}$ for some odd integer $t$, then $x$ is also a primitive vector of $L$. The result then follows since $c$ is a spinor exceptional integer (thus, a primitive spinor exceptional integer) for gen $\left(L^{\prime}\right)$.

To complete the proof, we turn to the technical details of the construction of a sublattice $K$ of $L_{2}$ for which $\theta(K, c)=\left(N_{c}\right)_{2}$ and $c \in Q^{*}(K)$. The sublattice $L^{\prime}$ of the previous paragraph is then produced as that global lattice on the space $\boldsymbol{Q} L$ satisfying

$$
\left(L^{\prime}\right)_{p}= \begin{cases}L_{p} & \text { if } p \text { is odd } \\ K & \text { if } p=2\end{cases}
$$

Of course, we need only consider the case of an integer $c$ for which $\theta\left(L_{2}, c\right) \neq\left(N_{c}\right)_{2}$. The possibilities for $c$ and the local structure of $L_{2}$ under which this can occur appear in the various cases and subcases of [11; Satz 4].

Consider first the case that $\operatorname{ord}_{2} c=0$. By surveying the subcases of case (a) (subcase (i)) and case (c) of [11; Satz 4] one sees that $\theta\left(L_{2}, c\right) \neq$ $\left(N_{c}\right)_{2}$ can occur only if $L$ has a 2 -adic splitting of the type $L_{2} \cong\left\langle b_{1}, 2^{r} b_{2}\right.$, $\left.2^{s} b_{3}\right\rangle$, say in $Z_{2}$-basis $\left\{u_{1}, u_{2}, u_{3}\right\}$, where $b_{1}, b_{2}$ and $b_{3}$ are 2-adic units, $r$ and $s$ are integers with $0 \leq r \leq s$, and $r \leq 4$. In fact, as $c \in Q^{*}\left(L_{2}\right)$, we may take $b_{1}=c$ in this splitting. Consider the sublattice $K=Z_{2} u_{1}+$ $Z_{2}\left(8 u_{2}\right)+Z_{2}\left(8 u_{3}\right)$ of $L_{2}$. Since $\theta\left(O^{+}(K)\right) \subseteq \theta\left(O^{+}\left(L_{2}\right)\right) \subseteq\left(N_{c}\right)_{2}$ and $c \in Q^{*}(K)$ by construction, it now follows from [11; Satz 4] that $\theta(K, c)=\left(N_{c}\right)_{2}$.

Finally, we consider the case that $\operatorname{ord}_{2} c=1$. If $\theta\left(L_{2}, c\right) \neq\left(N_{c}\right)_{2}$ for such an integer $c$, then the conditions of some subcase of case (a) (subcase (ii)) or case (b) of [11; Satz 4] must be satisfied. First, note that subcases $(\alpha)$ and ( $\gamma$ ) of case (a) (subcase (ii)) occur only when $\operatorname{ord}_{2} c \geq 2$. Also, subcases (i), (ii) and (iii) of case (b) fail to occur. In these three subcases $L_{2}$ must have the splitting $L_{2} \cong\left\langle b_{1}, 2^{r} b_{2}, 2^{s} b_{3}\right\rangle$ with $r$ even. So if $r \geq 2$, $L_{2}$ can primitively represent no element of $2 U_{2}\left(U_{2}\right.$ denotes the group of units of $Z_{2}$ ). If $r=0$, the cases are ruled out by explicit computation of 
the spinor norm groups $\theta\left(O^{+}\left(L_{2}\right)\right), \theta\left(O^{+}(K)\right)$ and $\theta\left(O^{+}\left(K^{\prime}\right)\right)$, with $K$ and $K^{\prime}$ as in the statement of [11; Satz 4]. To resolve subcase $(\beta)$ of case (a) (subcase (ii)), consider the required splitting of $L_{2}$ as $A(2,2) \perp\langle 2 b\rangle$, with $b \in U_{2}$, say in $Z_{2}$-basis $\left\{u_{1}, u_{2}, u_{3}\right\}$. As $c \in Q^{*}(A(2,2))$, the sublattice $K=Z_{2} u_{1}+Z_{2} u_{2}+Z_{3}\left(2 u_{3}\right)$ of $L_{2}$ has the desired properties. The last case to consider is that of subcase (iv) of case (b), where $L_{2}$ has the splitting $L_{2} \cong\left\langle b_{1}, 2 b_{2}, 2^{s} b_{3}\right\rangle$ in $Z_{2}$-basis $\left\{u_{1}, u_{2}, u_{3}\right\}$, with $b_{1}, b_{2}$ and $b_{3}$ in $U_{2}$ and $s$ an odd integer. If $x=\sum_{i=1}^{3} A_{i} u_{i}$ is a primitive vector of $L_{2}$ with $Q(x)=c$, then $A_{1} \in 2 Z_{2}$ and $A_{2} \in U_{2}$. So $c / 2$ is primitively represented by the lattice $\hat{L}_{2}$ obtained from $L_{2}$ by considering the quadratic mapping on $\hat{L}_{2}$ to be $\hat{Q}(x)=\frac{1}{2} Q(x)$ for $x \in L_{2}$ (i.e., $\hat{L}_{2}$ is the lattice $L_{2}$ scaled by $\frac{1}{2}$ in the terminology of [9]). As $\operatorname{ord}_{2}(c / 2)=0$, consideration of $\hat{L}_{2}$ reverts back to a case resolved previously. This exhausts all possible cases and completes the proof.

\section{REFERENCES}

[1] J. W. Benham and J. S. Hsia, On spinor exceptional representations, Nagoya Math. J., 87 (1982), 247-260.

[2] J. W. S. Cassels, Rational Quadratic Forms, Academic Press, London, 1978.

[ 3 ] —-, Rationale quadratische Formen, Jber. d. Dt. Math.-Verein, 82 (1980), 81-93.

[4] A. G. Earnest, Congruence conditions on integers represented by ternary quadratic forms, Pacific J. Math., 90 (1980), 325-333.

[ 5 ] J. S. Hsia, Representations by spinor genera, Pacific J. Math., 63 (1976), 147-152.

[6] B. W. Jones, Exceptional ternary and binary forms, preprint.

[ 7 ] - and G. L. Watson, On indefinite ternary quadratic forms, Canad. J. Math., 8 (1956), 188-194.

[8] M. Kneser, Darstellungsmasse indefiniter quadratischer Formen, Math. Z., 77 (1961), 188-194.

[9] O. T. O'Meara, Introduction to Quadratic Forms, Springer-Verlag, Berlin-Heidelberg-New York, 1963.

[10] M. Peters, Darstellungen durch definite ternäre quadratische Formen, Acta Arith., 34 (1977), 57-80.

[11] R. Schulze-Pillot, Darstellung durch Spinorgeschlechter ternärer quadratischer Formen, J. Number Theory, 12 (1980), 529-540.

[12] - private communication.

[13] C. L. Siegel, Indefinite quadratische Formen und Funktionentheorie I, Math. Ann., 124 (1951), 17-54.

[14] G. L. Watson, Integral Quadratic Forms, Cambridge University Press, Cambridge, 1960 .

Department of Mathematics

Southern Illinois University

Carbondale, Illinois 62901

USA 